The authors therefore corsider a uniformly rotating star of uniform density and perform a calculation of the energy radiated away in the form of gravitational waves during the collapse to lowest order approximation in the gravitational potential. This sort of collapse is known to occur for a white dwarf star with a mass a little in excess of the mass of the sun. Calculations based on Newtonian theory show that a spheroidal shaped object (such as might be the shape of a rotating white dwarf) becomes more oblate as the collapse proceeds, until finally, after a finite period of time, it assumes the unlikely shape of a zero thickness pancake at a minimum radius, say $r_{\text {min }}$. The Newtonian approximation will not be valid if $r_{\min }$ becomes comparable with the Schwarzschild radius $r_{\mathrm{sen}}$.

Thuan and Ostriker first find that a non-rotating spheroid of mass $M$ which starts at rest at infinity with infinitesimal eccentricity, emits a pulse of gravitational radiation of total energy $37 \times 10^{-3} \quad\left(r_{\mathrm{sch}} / r_{\min }\right)^{7 / 2} M c^{2}$ erg. This result should be compared with the analogous case of two point masses collapsing in from infinity from an initial state of rest which liberates only $1.2 \times 10^{-3}\left(r_{\mathrm{sch}} / r_{\min }\right)^{7 / 2} M c^{2}$ erg. Evidently spheroidal collapse is extremely efficient for the production of energy in this form.

Next the authors include the effects of rotation. The highest output would occur in this case if the star was very rapidly rotating with a large ratio of initial to final radius. Under these circumstances the energy radiated is boosted further to $109 \times 10^{-3}\left(r_{\mathrm{sch}} /\right.$ $\left.r_{\min }\right)^{7 / 2} M c^{2}$ - a gain of 91.6 on the value for two collapsing point masses. Moreover, as the authors point out, rapidly rotating stars are unstable to the growth of non-axisymmetrical perturbations, such as might be produced in reality by an oblique magnetic field. These additional disturbances would increase the radiation emitted still further. Physically, the reason why rapidly rotating collapsing stars radiate so much energy away in gravitational waves is partly due to the way in which the rotation delays the collapse of the equatorial parts of the star in a region of high gravitational potential during which time the poles quickly collapse inwards, so flattening the object still further.

Turning their attention to a realistic model of a white dwarf, the authors then estimate the energy emitted during the collapse of a $1.4 M \odot$ Maclaurin spheroid with uniform rotation and uniform density of $10^{9} \mathrm{~g} \mathrm{~cm}^{-3}$. An upper limit to this energy turns out to be about $10^{52} \mathrm{erg}$, and a lower limit around $10^{45} \mathrm{erg}$. A single pulse of this sort will have Fourier components nearly constant over a wide frequency range, after which they fall rapidly.

In practice a collapsing white dwarf might well emit a whole series of pulses as it 'bounced' back and forth through the pancake configuration, finally

\section{Still no gravitational radiation}

\section{by John Gribbin}

LEVINE and Garwin report in the latest issue of Physical Review Letters (33, 794-797; 1974) a new negative result for the detection of gravitational radiation pulses, at a level six times more sensitive than the experiments they reported last year (Phys. Rev. Lett., 31, $173-176 ; 1974)$. They say that they believe "the present results to be in substantial conflict with the detections reported by Weber".

The improved sensitivity over earlier results from the same group has been achieved chiefly through increasing the mass of the aluminium cylinder used as an antenna from $120 \mathrm{~kg}$ to $480 \mathrm{~kg}$; the resonance frequency is now $1,637 \mathrm{~Hz}$, close to that of Weber's apparatus, 1,661 $\mathrm{Hz}$. During the 27-day period December 3 to December 30, 1973, only one measurement out of $4.1 \times 10^{7}$ values obtained for the mean oscillation energy $k T$ contains a pulse substantially above the noise level.

According to Levine and Garwin, that pulse could be a gravity wave pulse, stress relaxation in the antenna, electrical pickup of "an intense but infrequent laboratory tone", or a signal-processor defect. Since no coincident pulse was observed at the University of Rochester detector, the gravitational radiation explanation seems the least tenable.

Further analysis of the dataincluding Weber's observations as far as they are available-seems to rule out both the possibility that gravity waves were detected in May 1973 but the pulses have not been produced since then, and the possibility that "hundreds of gravity waves were incident per day even during December 1973, giving our bar $<0.3 k T$ ". In addition, Levine and Garwin note that an error in the computer program used by Weber's group for some of the analysis would have produced a spuriously high number of pulses, and that Weber has acknowledged the existence of this error. All in all, it seems that gravitational radiation has not yet been detected. settling down into a neutron star. However, assuming a Weber-type experiment attempts to detect a single pulse of the type considered, even under the most favourable circumstances the collapsing star would still need to be as close as 100 light years for any hope of success

\section{The changing micrometeoroid influx}

\section{from David W. Hughes}

THE disparity between the flux of micrometeorites at $1 \mathrm{AU}$ obtained from lunar microcrater counts and from satellite data has been known for many years. A typical example is shown on page 380 , the satellite data coming from the work of Alexander (Baylor University, Waco, Texas), the data from the study of microcraters on the surface of lunar rocks and spherules coming from the work of Neukum and Schneider (Max-Planck-Institut für Kernphysik, Heidelberg). In the $10^{-10} \mathrm{~g}$ region the two techniques give results which differ by a factor of 200 and it is of great importance to decide if this difference is due to some physical cause or is just an unresolved systematic error in one or both techniques. Fortunately the influx of meteoroids to the lunar surface is the same as that to the Earth's upper atmosphere (most of the satellite observations are made in near-Earth space), the circumterrestrial dust cloud, thought in the late 1960 s to exist, now being discounted as a figment of incorrect callibration. But satellite-borne detectors do measure the present day influx, whereas lunar microcrater counts measure the mean influx over the last few tens of thousands of years.

To convert the crater population on a given lunar sample into an interplanetary dust flux the size and form of the craters have to be related to the mass of the causative meteoroid. One immediate problem is that crater size also depends on meteoroid velocity, dens:ty, and shape. Up to now the meteoroid impact velocity has been thought to be about $20 \mathrm{~km} \mathrm{~s}^{-1}$. Two recent results indicate that it is much lower. Doppler shift observations of the solar spectral lines scattered by the solar system dust cloud give a geocentric velocity of about $5 \mathrm{~km} \mathrm{~s}^{-1}$ and this observation has recently been backed up by measurements of microcraters found in glass, steel and pyroxene surfaces exposed for 46 days during the Skylab mission. Nagel, Fechtig, Schneider and Neukum (Max-Planck-Institut, Heidelberg) reported these observations at the recent COSPAR Meeting (Sao Paulo, 1974). They compared the spall zones, smoothness of the central depression, fracture damage and general shape of the 47 observed craters (diameters $>1 \mu \mathrm{m}$ ) 